\title{
Dopamine receptor 4 promoter polymorphism modulates memory and neuronal responses to salience
}

\author{
B.A. Strange N. Gartmann J. Brenninkmeyer J. Haaker A. Reif R. Kalisch C. Büchel
}

\begin{abstract}
A B S T R A C T
Animal models and human functional imaging data implicate the dopamine system in mediating enhanced encoding of novel stimuli into human memory. A separate line of investigation suggests an association between a functional polymorphism in the promoter region for the human dopamine 4 receptor gene (DRD4) and sensitivity to novelty. We demonstrate, in two independent samples, that the $-521 \mathrm{C}>\mathrm{T} D R D 4$ promoter polymorphism determines the magnitude of human memory enhancement for contextually novel, perceptual oddball stimuli in an allele dose-dependent manner. The genotype-dependent memory enhancement conferred by the $\mathrm{C}$ allele is associated with increased neuronal responses during successful encoding of perceptual oddballs in the ventral striatum, an effect which is again allele dose-dependent. Furthermore, with repeated presentations of oddball stimuli, this memory advantage decreases, an effect mirrored by adaptation of activation in the hippocampus and substantia nigra/ventral tegmental area in C carriers only. Thus, a dynamic modulation of human memory enhancement for perceptually salient stimuli is associated with activation of a dopaminergic-hippocampal system, which is critically dependent on a functional polymorphism in the DRD4 promoter region.
\end{abstract}

\section{Introduction}

Dopamine activity signals unexpected, salient, motivationallyrelevant information (Berridge, 2007; Horvitz, 2000; Ljungberg et al., 1992; Redgrave et al., 1999; Ungless, 2004). Enhanced memory for novel, or salient, stimuli is thought to be mediated by dopamine via hippocampal inputs from the dopaminergic midbrain (Lisman and Grace, 2005), which comprises the substantia nigra and ventral tegmental area (SN/VTA) (Duzel et al., 2009). In humans, processing of unexpected, salient stimuli can be studied using "oddball" paradigms, in which the oddball stimulus deviates from its prevailing context along a particular dimension. Long-term human memory for these contextually novel stimuli is enhanced (Ranganath and Rainer, 2003), an effect abolished by hippocampal damage (Kishiyama et al., 2004). A role for dopamine in contextual novelty detection is suggested by human functional magnetic resonance imaging ( $\mathrm{FMRI}$ ) studies demonstrating activation in dopaminergic areas in response to novel oddball stimuli (Bunzeck and Duzel, 2006; Duzel et al., 2009).

Both animal and human data point towards a critical role for the dopamine 4 (D4) receptor in novelty processing. Drd4 gene knockout mice show decreased exploration in response to novel stimuli (Dulawa et al.,
1999; Rubinstein et al., 1997), whereas a selective D4 receptor agonist increases novel object exploration in mice (Powell et al., 2003). The D4 receptor is preferentially expressed in limbic regions, including the hippocampus (Mrzljak et al., 1996), prefrontal cortex (Ariano et al., 1997; Defagot et al., 1997; Matsumoto et al., 1995), and globus pallidus (Mrzljak et al., 1996). Although D4 receptor expression has been shown in primate $\mathrm{SN}$, this is in SN pars reticulata (Mrzljak et al., 1996) and not the SN pars compacta or VTA, which contain the majority of dopaminergic neurones in the SN/VTA (Duzel et al., 2009). D4 receptor expression in SN pars compacta has, however, been shown in rodents (Defagot et al., 1997). In humans, the rather small (3.5 kb) DRD4 gene has generated much interest due to a high degree of functional variability, and an observed association between functional DRD4 polymorphisms and attention deficit/hyperactivity disorder (ADHD) (Franke et al., 2011), substance abuse (Li et al., 2011) and the personality trait of novelty seeking (NS) (Munafo et al., 2008b), which is considered a characteristic feature of both disorders.

Human NS is characterized by impulsivity, exploratory drive and excitability, and has been proposed to reflect individual differences in dopamine system sensitivity (Cloninger et al., 1993). Although initial evidence suggested an association between NS and a variable number tandem repeat (VNTR) polymorphism in exon III of DRD4 (Benjamin et al., 1996; Ebstein et al., 1996), a recent meta-analysis (Munafo et al., 2008b) refutes this, but demonstrates strong evidence for an association between NS and a cytosine to thymine polymorphism in the DRD4 promoter region ( $-521 \mathrm{C}$ T single nucleotide polymorphism, SNP). For this 
promoter polymorphism, which is in linkage disequilibrium with the exon III VNTR (Ekelund et al., 2001; Strobel et al., 2002), the C allele is associated with increased transcription levels of up to $40 \%$ compared with the T allele in vitro (Okuyama et al., 1999). However, in human post-mortem brain the $-5210>$ T SNP was not found to significantly affect DRD4 mRNA expression levels (Simpson et al., 2010), thus whether this SNP is functional is currently not clear.

In animal models of novelty seeking, both striatal dopamine (Blanchard et al., 2009) and the hippocampus (Blanchard et al., 2009; Kabbaj et al., 2000) are implicated in behavioral responses to novelty. Thus, given the overlap in brain areas involved in both enhanced memory for novel stimuli and novelty seeking, and the common proposed role for dopamine in these processes, we hypothesized that memory for contextually novel stimuli in humans is modulated by DRD4 promoter polymorphism. We tested this hypothesis in healthy human subjects, pre-selected on the basis of DRD4 genotype. Although our experiments address the role of the promoter $-521 \mathrm{C}>\mathrm{T}$ SNP in novelty-evoked memory enhancement, in view of the (albeit less consistent) evidence for an association between DRD4 exon III VNTR and NS (Munafo et al., $2008 \mathrm{~b}$ ) subjects were also stratified on the basis of this polymorphism to enable the VNTR to be included as a covariate in our statistical model.

Subjects encoded lists of neutral nouns during fMRI scanning (Fig. 1a). The critical manipulation was the presentation of a perceptual ( $P$ ) oddball (a noun presented in a novel font) and an emotional (E) oddball (aversive noun) in each list. After encoding each list, subjects performed a distractor task followed by free recall. Both recall performance, and evoked neuronal responses, for oddball nouns were compared to neutral control nouns randomly selected from the same list. We demonstrate that the DRD4 promoter polymorphism significantly modulates memory for perceptual oddballs in an allele dose-dependent manner, with $\mathrm{C} / \mathrm{C}$ homozygotes showing approximately twice the memory advantage for these stimuli than T/T subjects. This effect is observed again in a larger, independent sample of individuals completing the same behavioral task outside of fMRI scanning. In the scanned group, this memory enhancement is associated with a $C$ allele-dependent enhanced activation of dopaminergic areas and hippocampus during successful encoding of perceptual oddballs.

\section{Materials and methods}

\section{Samples}

Sample sizes are in accord with power considerations for determining genetic contributions to episodic memory in humans (Rasch et al., 2010a).

\section{fMRI scanning}

From a pool of right-handed, male, native German speaking subjects, 50 subjects (age range, 19-45; mean, 27.4 years) were selected on the basis of DRD4 genotype ( $-5210 \mathrm{~T}$ and exon III VNTR) to take part in this study. Three subjects were rejected due to technical failure.

\section{Behavioral replication}

From a second pool of right-handed, male and female native German speaking subjects, 109 subjects were selected on the basis of $-521 \mathrm{C}>\mathrm{T}$ DRD4 genotype. For this sample, subjects were not pre-selected by exon III VNTR. For inclusion of this polymorphism as a covariate in our statistical models, we divided subjects on the basis of the presence of one or more long versions of the VNTR (i.e., one or more 7- or 8-repeat VNTR alleles). Eleven subjects were rejected due to poor performance on the shallow encoding task (see below). Thus, 98 subjects ( 48 female) were entered into statistical analyses (age range, 19-46; mean, 26.5 years).

In both samples, all subjects are of self-reported German ethnicity. All are white, were born in Germany, with German as mother tongue and nationality, and self-report having both white parents and grandparents. All subjects gave informed consent and were free of neurological or psychiatric history. Subjects and experimenter were blind to genotype. The study had full ethical approval.

\section{Genotyping}

For fMRI scanning and behavioral replication samples, peripheral venous blood was drawn from all volunteers, and genomic DNA was extracted from blood using a routine de-salting method.

\section{Primary protocol}

DRD4 $-5210 \mathrm{~T}$ ( rs1800955) and exon III VNTR were genotyped by PCR using standard protocols. Briefly, $-5210 \mathrm{~T}$ was genotyped using the primers 5'-CGGGGGCTGAGCACCAGAGGCTGCT-3' (forward) and 5'-GCATCGACGCCAGCGCCATCCTACC- $3^{\prime}$ (reverse). Following an initial 5 min of denaturation at $95^{\circ} \mathrm{C}, 32$ PCR cycles were performed before a final 5 min step at $72^{\circ} \mathrm{C}$. Amplicons were then digested with FspI and visualized with agarose gel electrophoresis. Genotyping of DRD4 exon III VNTR was performed using Vent polymerase (New England Biolabs) and a high denaturing temperature $\left(95^{\circ} \mathrm{C}\right.$ for $\left.5 \mathrm{~min}\right)$ with a combined annealing and extension reaction for $10 \mathrm{~min}$ at $72^{\circ} \mathrm{C}$. The primers were 5'-GCGACTACGTGGTCTACTCG- $3^{\prime}$ and 5'-AGGACCCTCATGGCCTTG- $3^{\prime}$. Thirty PCR cycles were performed and subsequently, the reaction mixture was electrophoresed on a $3.5 \%$ agarose gel with ethidium bromide.

\section{Secondary protocol}

To replicate the genotyping results, a second method was applied to determine rs1800955 genotype using TaqMan-based genotyping. Genotyping was carried out in a volume of $10 \mathrm{ml}$ containing $10 \mathrm{ng}$ of genomic DNA, $5 \mu \mathrm{l}$ of ABgene Mastermix (ABgene Ltd., Hamburg, Germany), and $0.5 \mu \mathrm{l}$ of the Taqman assay (assay ID: Taqman assay: C_7470700_30; Applied Biosystems, Darmstadt, Germany). Amplification was performed on a Biorad CFX 384 Real-Time PCR System (Biorad, Munich, Germany) starting with $10 \mathrm{~min}$ at $95^{\circ} \mathrm{C}$, followed by 50 cycles of $15 \mathrm{~s}$ at $92^{\circ} \mathrm{C}$ and $1 \mathrm{~min}$ at $60^{\circ} \mathrm{C}$. Genotypes were scored using the software supplied by the manufacturer.

Tertiary protocol (run where primary and secondary were discrepant)

An alternative RFLP protocol for detection of SNP rs1800955 was run in the case that the primary and secondary protocols yielded discrepant findings. A 276-bp PCR product was amplified using the following reaction mix: $50 \mathrm{ng}$ of genomic DNA in $75 \mathrm{mM}$ Tris- $\mathrm{HCl}$ (pH9.0), $20 \mathrm{mM}$ ammonium sulfate, $0.01 \%$ Tween $20,2.7 \mathrm{mM}$ magnesium chloride, $0.4 \mu \mathrm{M}$ of each of the primers, forward ( $5^{\prime}$-GTCCGCCCAGTTTCGGAGGC- $3^{\prime}$ ) and reverse (5'-AAACCTCCCTCCCAGGCCCG-3'), $0.1 \mathrm{mM}$ dNTP, and $1 \mathrm{U}$ Taq polymerase. After an initial denaturation for 5 min at $95^{\circ} \mathrm{C}, 35$ cycles of denaturation at $95^{\circ} \mathrm{C}$ for $40 \mathrm{~s}$, annealing at $62^{\circ} \mathrm{C}$ for $40 \mathrm{~s}$ and extension of $72{ }^{\circ} \mathrm{C}$ for $40 \mathrm{~s}$ were performed, followed by a final extension of $72{ }^{\circ} \mathrm{C}$ for $5 \mathrm{~min}$. PCR products were digested with BssHII at $50^{\circ} \mathrm{C}$ for $2 \mathrm{~h}$. PCR were visualized on a $3.5 \%$ agarose gel containing ethidium bromide. The undigested PCR product carries the T variant, whereas the digested product with two fragments of $181 \mathrm{bp}$ and $95 \mathrm{bp}$ contains the $\mathrm{C}$ allele.

For the scanned sample, the allelic distribution of both genotypes of interest (DRD4 $-521 \bigcirc \mathrm{T}$ promoter SNP and DRD4 exon III VNTR) was: 17 T/T homozygote subjects (9 DRD4 exon III VNTR 4/4; 8 4/7), $20 \mathrm{C} / \mathrm{T}$ heterozygotes $(104 / 4 ; 104 / 7)$ and $10 \mathrm{C} / \mathrm{C}$ homozygotes $(64 / 4 ; 44 / 7)$. For the replication group, the allelic distribution of the DRD4 - 521C $\mathrm{T}$ promoter SNP and DRD4 exon III VNTR was: $34 \mathrm{~T} / \mathrm{T}$ homozygote subjects (27 DRD4 exon III VNTR long absent; 7 long present), $34 \mathrm{C} / \mathrm{T}$ heterozygotes (21 long absent; 13 long present) and $30 \mathrm{C} / \mathrm{C}$ homozygotes (21 long absent; 9 long present). The two polymorphisms were in linkage disequilibrium (scanned sample $D^{\prime}=-0.11$; replication sample $D^{\prime}=0.22$ ). For additional demographics, see Supplementary Tables 1 and 2. The distribution of DRD $4-521 \bigcirc \mathrm{T}$ promoter alleles, in the 1597 individuals comprising the pools from which our subjects were selected, was in Hardy-Weinberg equilibrium $\left(\chi^{2}=0.250 ; P=0.617\right)$. No other 
a
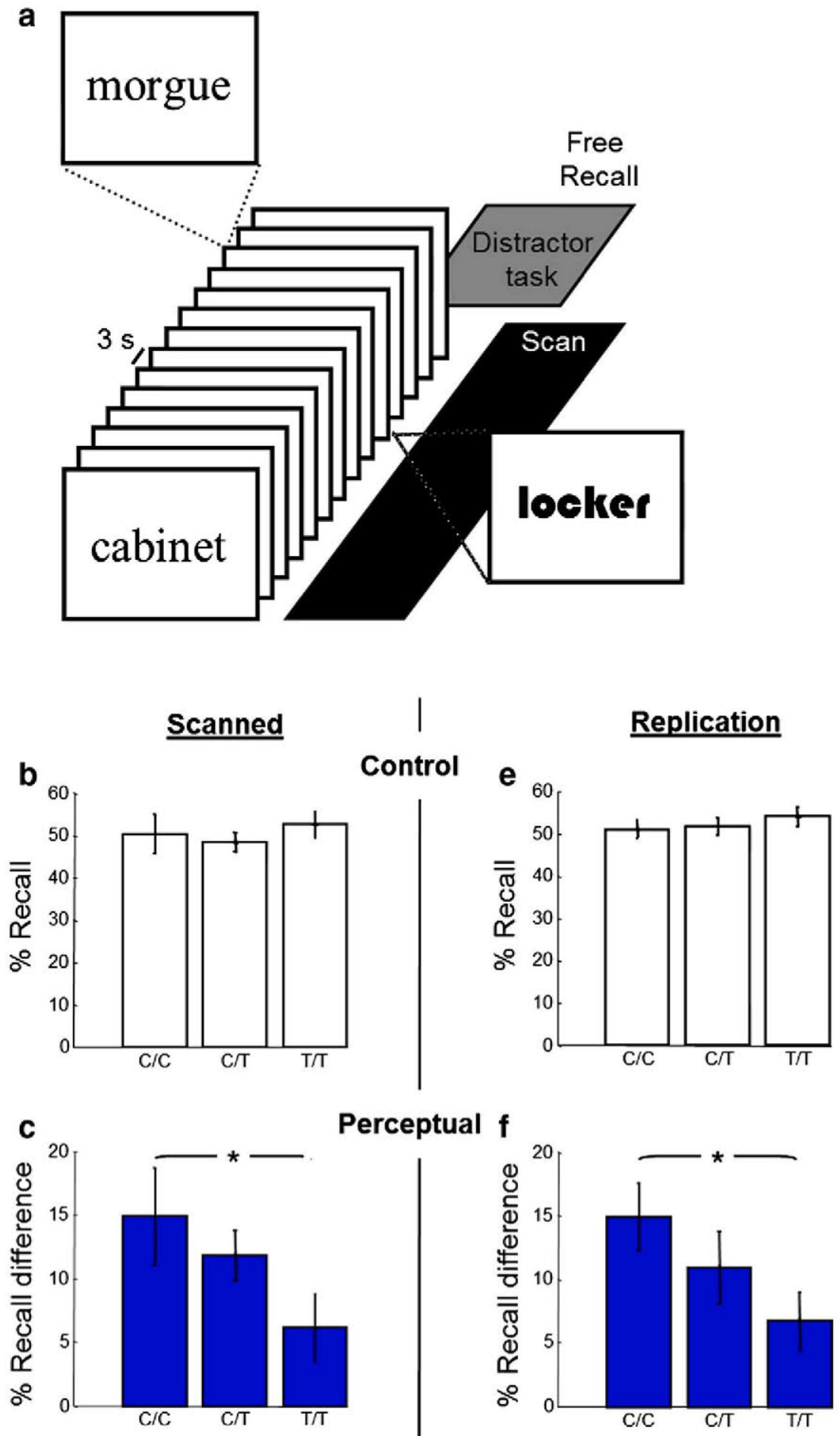

Perceptual f
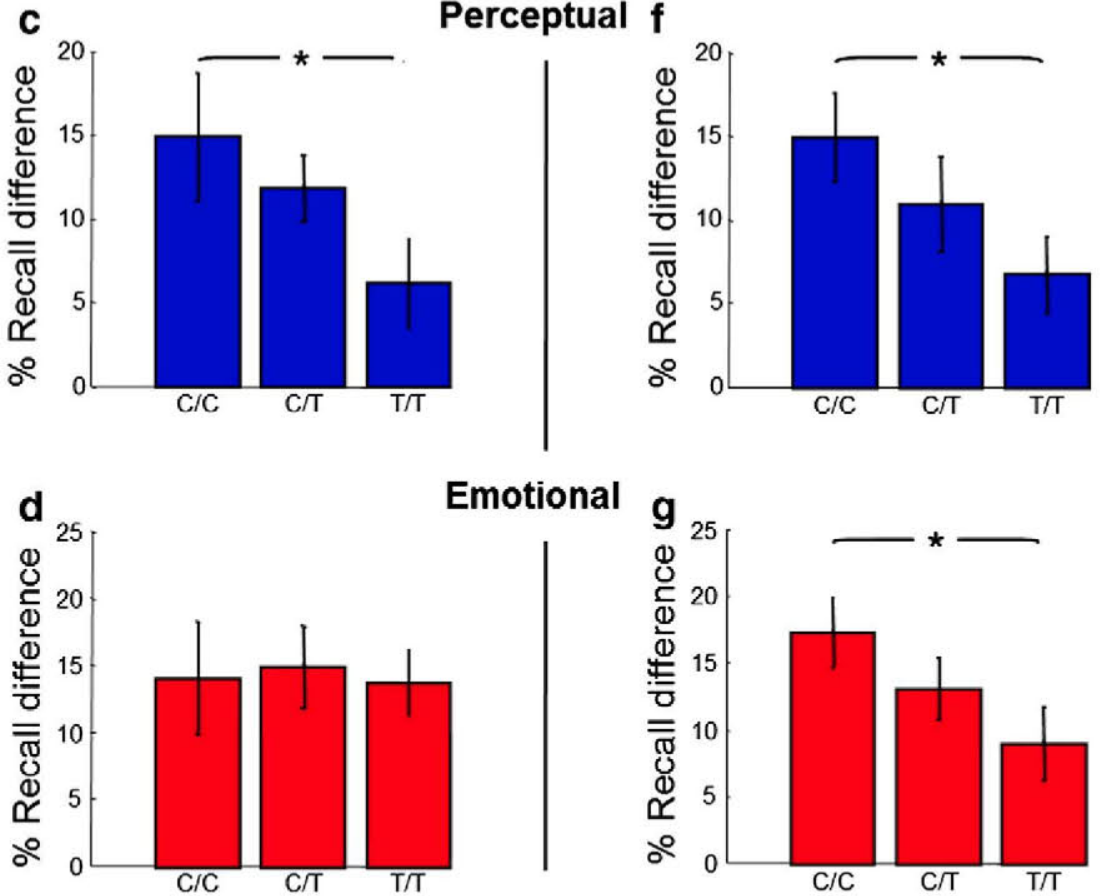

Emotional

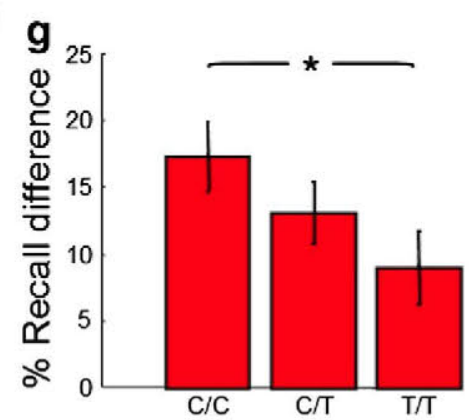


polymorphisms besides the DRD4 $-521 \mathrm{C}$ T promoter SNP and DRD4 exon III VNTR were examined.

\section{Personality scales}

\section{fMRI scanning and behavioral replication samples}

All subjects completed Cloninger's Temperament and Character Inventory (TCI), a questionnaire containing 240 dichotomous variables to measure seven personality factors: novelty seeking, reward dependence, harm avoidance, persistence, cooperativeness, self-directedness and self-transcendence (Cloninger, 1994). Novelty seeking comprises measures of exploratory excitability, impulsiveness, extravagance and disorderliness.

Task

\section{fMRI scanning}

Subjects were scanned while viewing nouns presented visually at a rate of one every $3 \mathrm{~s}$ (stimulus duration, $1 \mathrm{~s}$ ). Subjects were presented with 34 lists of 14 German nouns, identical to our previous experiments (Strange et al., 2003). 20 s after scanning onset, the start of each list was prompted by the presentation of the words "Neue Liste" (New List). $6 \mathrm{~s}$ after the presentation of the 14th word of each list, a number was presented indicating the onset of a $30 \mathrm{~s}$ distraction task during which subjects were instructed to count backwards in threes (out loud) from the presented number. To eschew artifacts arising from verbalization during fMRI acquisition, scanning terminated following presentation of this number. The distractor task was followed immediately by instructions to free-recall the words presented in the preceding list. The entire procedure was repeated for the 34 lists.

All 14 nouns in each list were of the same semantic category. Of these, 12 nouns were emotionally neutral and presented in the same font (control nouns). The first five nouns in each list were always control nouns. A perceptual oddball was presented in a different font but was emotionally neutral. An emotional oddball was aversive in content but perceptually identical to neutral nouns. The order of list presentation, as well as the order of noun presentation within-list, was randomized. Nouns were presented in Times font ( 48 point; $4-10^{\circ}$ of horizontal visual angle) except for perceptual oddballs, which appeared in 17 different fonts (Fig. 1a). These fonts were repeated in the second half of the experiment.

Subjects were instructed to indicate with a push-button whether or not the first letter in the noun (always capitalized in German) contained a curve (shallow encoding). It was emphasized that they were not to adopt mnemonics such as imagery or making sentences or stories. Encoding instructions were provided visually at the start of the experiment. Recall performance is expressed relative to two randomly selected neutral nouns (one for each oddball type) presented at encoding. These control nouns, like oddballs, could not occur within the first five nouns of each list and could not immediately follow an oddball or another chosen control noun.

\section{Behavioral replication}

The psychological task for the replication sample outside of fMRI scanning was identical, except that, in view of the rapid adaptation of $P$ noun memory enhancement in the scanned sample, we only considered the first 17 word lists in which each oddball is presented in a unique font. A number of subjects in this sample failed to make pushbutton responses to each word, thus we excluded subjects whose performance on the shallow memory task was less than a threshold of $80 \%$ correct on control nouns.

\section{Imaging}

A 3 T Siemens TRIO system (Siemens, Erlangen, Germany) with a standard 12 channel head coil was used to acquire both highresolution T1-weighted anatomical images and gradient-echo echoplanar (EPI) T2*-weighted MRI image volumes with blood oxygenation level-dependent contrast. For each studied list, 24 EPI volumes were acquired, plus five additional volumes, acquired at the start of each session and subsequently discarded, to allow for T1 equilibration effects. Each volume comprised 38 axial slices ( $2 \mathrm{~mm}$ thick; repetition time $2.47 \mathrm{~s}$; echo time $30 \mathrm{~ms}$ ). Each imaging time series was then realigned to correct for interscan movement, slice-time corrected, normalized into a standard anatomical space, and smoothed with a Gaussian kernel of $8 \mathrm{~mm}$ full width half-maximum.

\section{Data analysis}

Imaging data were analyzed using statistical parametric mapping (SPM5; http://www.fil.ion.ucl.ac.uk/spm) employing an event-related model with a two-stage random effects procedure. To test for subsequent memory effects, we specified eight effects of interest: the events corresponding to $\mathrm{E}$ and $\mathrm{P}$ noun presentation and the two randomly selected control nouns, separated according to whether they were later recalled or forgotten. Trial-specific responses were modeled by convolving a delta function that indicated each event onset with a canonical hemodynamic response function (HRF) to create regressors of interest. Each list was modeled separately. Encoding events for which there were absent or incorrect responses, as well as events corresponding to all other nouns, the presentation of the "New List" marker, and the number to indicate onset of the distractor task, were modeled as regressors of no interest (total of 3). Six movement parameters were modeled as nuisance covariates.

Session-specific parameter estimates of the magnitude of the hemodynamic response for each stimulus type were calculated for each voxel in the brain. A contrast of parameter estimates modeling each comparison of interest (e.g., remembered $v s$. forgotten perceptual $v s$. control nouns) was calculated in a voxel-wise manner to produce, for each subject, one contrast image for that particular effect. For the random effects analysis, each subject's contrast image was entered into a general linear model (GLM) across subjects. The covariate of interest in this model was an allele dose-dependent contrast for $-5210 \mathrm{~T}$ DRD4 promoter genotype based on perceptual noun memory enhancement ( $\mathrm{C} / \mathrm{C}$ vs. $\mathrm{C} / \mathrm{T}$ vs. T/T homozygotes). VNTR $(4 / 4,4 / 7)$ genotype was included as a nuisance covariate. Identical analyses were then performed for emotional nouns. For conjunction analyses, the GLM included a contrast image of response to each oddball type, as well as covariates for $-521 C>\mathrm{T} D R D 4$ promoter and VNTR genotype. Conjoint effects were tested against the global null hypothesis (Friston et al., 2005). Conjunction analyses require an independent baseline for each effect being tested, thus two control nouns were randomly assigned as the control for each oddball type.

We carried out a small volume correction (SVC) to the P values of the ensuing maxima on all regions which pertain to the animal model of novelty-evoked memory enhancement (Lisman and Grace, 2005). Thus, we report responses at a threshold of $P<0.05$, family-wise corrected for a spherical search volume of $20-\mathrm{mm}$ diameter centered on previously reported $x, y, z$ coordinates $( \pm 12,8,-8)$ for the ventral striatum (Zink et al., 2003) and (28, $-20,-14)$ for the right hippocampus (Yamaguchi et al., 2004), and a spherical search volume of $10-\mathrm{mm}$ diameter centered on $( \pm 8,-14,-8)$ for the SN/VTA (Guitart-Masip et al., 2010). Activations outside of these regions that survive an

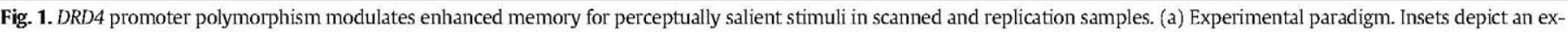

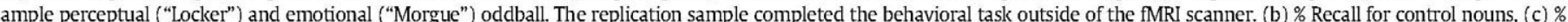

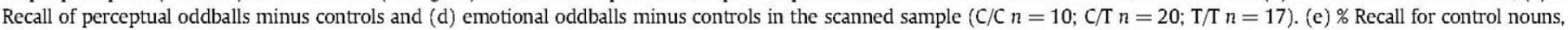

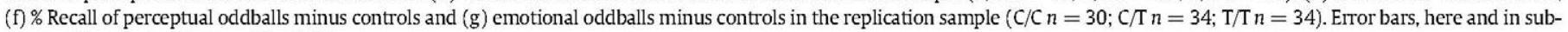
sequent figures, depict s.e.m. ${ }^{*} P<0.05$. 
uncorrected height threshold of $P<0.001$ and extent threshold of 10 contiguous voxels are presented in Tables 1-3.

We consider activation of the entire SN/VTA complex rather than individual components because in humans, mesolimbic and mesocortical dopaminergic projection systems are dispersed throughout the SN/VTA complex (Duzel et al., 2009).

\section{Results}

Behavior

\section{Perceptual oddball memory}

In the scanned sample, which included only male subjects, $-5210 \mathrm{~T}$ DRD4 promoter polymorphism does not affect memory for control nouns (Fig. 1b; one-way ANOVA for control noun recall across genotype $\left.F_{2,44}=0.542 P=0.585\right)$. All $-521 \bigcirc \mathrm{T}$ genotypes show memory enhancement for P oddballs (Fig. 1c). Critically, and according to our prediction, the DRD4 promoter polymorphism significantly modulates enhanced memory for P oddballs. For the 34 lists viewed by the scanned sample (Fig. 1c), we observe a $C$-allele dose-dependent effect on $P$ noun memory enhancement. A univariate ANOVA with [P-control noun] recall as the dependent variable and the number of $C$ alleles in the promoter SNP as a covariate reveals a significant effect of the promoter polymorphism $F_{1,45}=5.113, P=0.029, \eta^{2}=0.0519$. A one-sample $t$-test on [P-control noun] recall in the T/T group confirmed that, although of smaller magnitude than $\mathrm{C} / \mathrm{C}$ and $\mathrm{C} / \mathrm{T}$ groups, T/T subjects show enhanced memory for P oddballs $(t(16)=2.282 P=0.037$, two-tailed).

To demonstrate that the effect of the DRD4 promoter SNP on perceptual oddball memory is independent of the VNTR polymorphism, we repeated the univariate ANOVA on [P-control noun] recall with promoter genotype as covariate and VNTR polymorphism $(4 / 4,4 / 7)$ as nuisance covariate. This analysis demonstrated a significant effect of the promoter polymorphism $F_{1,44}=5.056, P=0.030$, and no effect of the VNTR polymorphism $F_{1,44}=0.087, P=0.770$. Furthermore, the reported association between DRD4 genotype and NS personality trait (Munafo et al., 2008b) raises a possibility that differential P noun memory enhancement is attributable to NS rather than $-521 \mathrm{C}>\mathrm{T}$ DRD4 promoter polymorphism. Thus, to ensure that the genotype-dependent memory effects we report are independent of NS, we also performed a univariate ANOVA on [P-control noun] recall with promoter genotype and NS $T$-value as covariates (NS T-values are shown in Supplementary Fig. 1). This again confirmed a significant effect of promoter polymorphism $F_{1,44}=5.385, P=0.025$, and no effect of NS score $F_{1,44}=0.553, P=0.461$.

\section{Emotional oddball memory}

Emotionally aversive oddball nouns can be considered as salient and motivationally relevant. Animal studies demonstrate increased activity

\section{Table 1}

Local maxima for subsequent memory effects for $\mathrm{P}$ oddballs in step-wise $\mathrm{C}$ allele dosedependent contrast $(\mathrm{C} / \mathrm{C}>\mathrm{C} / \mathrm{T}>\mathrm{T} / \mathrm{T})$. Also given is the corresponding activation in the cluster of interest for subsequent memory effects for P oddballs in a subset of C carriers vs. a subset of T/T homozygotes, matched for performance. Here, and in subsequent tables, we report only activations surviving correction for search volume, or an uncorrected threshold of $P<0.001$, with an extent threshold of 10 contiguous voxels. For the performance-matched analysis we report activation in cluster of interest at a threshold of $P<0.005$ uncorrected.

\begin{tabular}{lll}
\hline Brain area & MNI co-ordinates $(x, y, z)$ & Z-score \\
\hline Right ventral striatum & $16,8,-14$ & $4.22^{*}$ \\
Smoothing 6 mm FWHM & $18,8,-10$ & 3.36 \\
Left medial frontal gyrus & $-10,32,42$ & 3.68 \\
Left inferior temporal sulcus & $-48,-50,-4$ & 3.52 \\
Right amygdala & $28,-2,-16$ & 3.49 \\
Left lateral occipital sulcus & $-38,-86,0$ & 3.37 \\
& & \\
Repeat comparison in subsample of C carriers matched for performance & \\
Right ventral striatum & $20,6,-8$ & 3.08 \\
\hline
\end{tabular}

\footnotetext{
* $P<0.05$ family-wise corrected for small volume. FWHM: full-width half maximum.
}

Table 2

Local maxima for subsequent memory effects for P oddballs greater than control nouns in step-wise $C$ allele dose-dependent contrast.

\begin{tabular}{lll}
\hline Brain area & MNI co-ordinates & Z-score \\
\hline Left middle temporal gyrus & $-58,-18,-14$ & 3.68 \\
Left ventral striatum & $-14,18,-14$ & 3.67 \\
$\quad$ Smoothing 6 mm FWHM & $-16,20,-10$ & 3.65 \\
Bilateral ventral striatum & $0,10,-10$ & 3.66 \\
$\quad$ Smoothing 6 mm FWHM & $0,10,-10$ & 4.03 \\
Right ventral striatum & $4,10,-10$ & $3.46^{*}$ \\
Left ventral striatum & $-4,8,-10$ & $3.40^{*}$ \\
Left inferior temporal sulcus & $-56,-52,-12$ & 3.47 \\
& \\
Repeat comparison in subsample of C carriers matched for performance & \\
Left ventral striatum & $2,8,-10$ & 2.96 \\
\hline$* P<0.05$ family-wise corrected for small volume.
\end{tabular}

* $P<0.05$ family-wise corrected for small volume.

of dopamine cells in response to aversive stimuli (Matsumoto and Hikosaka, 2009), as well as a specific role for D4 receptors in emotional learning (Laviolette et al., 2005). We therefore next tested whether DRD4 promoter polymorphism effects in the scanned sample on memory extend to $\mathrm{E}$ oddballs, or are specific to P nouns. There was no clear genotype-dependent effect in the scanned sample (Fig. 1d). For recall of the 34 lists, a genotype $(C / C, C / T, T / T) \times$ noun type (Emotional oddball, control noun) $3 \times 2$ repeated measures ANOVA revealed a significant main effect of noun type $F_{1,44}=57.291 P<0.001$, but no significant genotype $\times$ noun type interaction $F_{2,44}=0.051 P=0.951$. Further, a univariate ANOVA for [E-control noun] recall as the dependent variable and the number of $C$ alleles in the promoter SNP as a covariate failed to show a significant effect of genotype $F_{1,45}=0.015, P=0.903$.

\section{Replication sample}

Our replication sample, which included male and female subjects, shows a strikingly similar pattern of DRD4 promoter polymorphismdependent memory enhancement for P oddball nouns. Again we demonstrate no effect of $-521 C>$ T DRD4 promoter polymorphism on memory for control nouns (one-way ANOVA $F_{2,95}=0.496 P=0.661$ ) in the replication sample (Fig. 1e). Critically, an allele dose-dependent effect of the DRD4 promoter-dependent modulation of perceptual noun memory for the 17 lists is again observed in the replication sample (Fig. 1f). A univariate ANOVA with [P-control noun] recall as the dependent variable and the number of $C$ alleles in the promoter SNP as a covariate revealed a significant effect of the promoter polymorphism $F_{1,96}=4.906$, $P=0.029, \eta^{2}=0.0322$. This effect remains significant when DRD4 VNTR polymorphism, NS T-values and gender are included as covariates of no interest. A one-sample $t$-test on [P-control noun] recall in the $\mathrm{T} / \mathrm{T}$ group confirmed that, although of smaller magnitude than $\mathrm{C} / \mathrm{C}$ and $\mathrm{C} / \mathrm{T}$ groups, $\mathrm{T} / \mathrm{T}$ subjects show enhanced memory for $\mathrm{P}$ oddballs $(t(33)=2.950 \mathrm{P}=$ 0.006 , two-tailed).

One notable difference between replication and scanned samples is an allele dose-dependent effect of $-521 C>\mathrm{T}$ DRD4 promoter

Table 3

Areas demonstrating a significant linearly decaying subsequent memory effect for P oddballs vs. control nouns in step-wise $C$ allele dose-dependent contrast.

\begin{tabular}{lll}
\hline Brain area & MNI co-ordinates & Z-score \\
\hline Right precuneus & $24,-52,22$ & 3.68 \\
Right hippocampus & $36,-20,-16$ & $3.35^{*}$ \\
Right SN/NTA & $4,-16,-8$ & $2.95^{*}$ \\
Smoothing 6mm FWHM & $6,-18,-6$ & 3.19 \\
& & \\
Repeat comparison in subsample of C carriers matched for performance & 4.58 \\
Right hippocampus & $38,-20,-14$ & 2.70 \\
Right SN/VTA & $6,-16,-8$ & \\
\hline
\end{tabular}

* $P<0.05$ family-wise corrected for small volume. 
polymorphism on E noun memory (Fig. 1g), analogous to that observed for Perceptual oddballs (univariate ANOVA for [E-control noun] recall and the number of $C$ alleles in the promoter SNP as a covariate $\left.F_{1,96}=5.235, P=0.024, \eta^{2}=0.0291\right)$. The genotype-dependent effects on $\mathrm{P}$ and $\mathrm{E}$ memory are of similar magnitude in the replication sample. A genotype $(\mathrm{C} / \mathrm{C}, \mathrm{C} / \mathrm{T}, \mathrm{T} / \mathrm{T}) \times$ word type (Emotional oddball, Perceptual oddball) $3 \times 2$ repeated measures reveals no significant interaction between memory for the two oddball types $\left(F_{1,95}=0.001 P=0.999\right)$. One possible explanation for the discrepancy between samples is that, in contrast to the scanned sample, the replication sample included female, as well as male, subjects. However, emotion-induced memory enhancement evoked by this task has been shown to be present in both genders (Strange et al., 2003), and there is no significant gender effect on the DRD4 promoter allele dose-dependent effect on emotional memory (genotype $(\mathrm{C} / \mathrm{C}, \mathrm{C} / \mathrm{T}, \mathrm{T} / \mathrm{T}) \times$ gender interaction for [E-control noun] recall $F_{1,94}=1.137 P=0.289$ ). A further discrepancy is that behavioral analysis for the scanned sample included 34 word lists, as opposed to 17 lists in the replication sample. To control for this possibility, we examined memory for emotional oddballs in the scanned group but taking only the first 17 lists presented. Again, the allele dose-dependent effect of the DRD4 promoter-dependent modulation of memory for $\mathrm{E}$ nouns was not observed (univariate ANOVA for [E-control noun] recall for the 1 st half of the scanned experiment as the dependent variable and the number of $C$ alleles in the promoter SNP as a covariate $F_{1,45}=$ $0.075, P=0.786)$.

\section{Imaging}

\section{Responses to perceptual oddballs}

Having established a consistent behavioral role for $-521 \mathrm{C}>\mathrm{T}$ DRD4 promoter polymorphism in enhanced memory for P nouns, we next examined the neuronal correlates of differential $\mathrm{P}$ oddball processing as a function of $C$ allele number in the scanned sample. To ensure that the effects we report are independent of the DRD4 exon III VNTR, this was included as a covariate in all analyses. Preliminary analyses comparing responses to $\mathrm{P} v$ s. control nouns, irrespective of whether nouns were subsequently freely recalled or forgotten, are presented in Supplementary Figs. 2 and 3. In "subsequent memory" analyses, we compared encoding-related neuronal responses to Perceptual oddball nouns that were subsequently freely recalled at the end of each distractor task vs. those forgotten for all 34 lists. A P noun subsequent memory effect is significantly enhanced by the DRD4 promoter C allele, in an allele dose-dependent manner, in the right ventral striatum and amygdala (Figs. 2a-c; Table 1). The plot in Fig. 2d demonstrates increased activation in the right ventral striatum for subsequently recalled $(\mathrm{R}) v$ s. forgotten ( $\mathrm{F}$ ) P nouns in $\mathrm{C} / \mathrm{C}$ homozygotes, which decreases with the number of $\mathrm{T}$ alleles. Subsequent memory-related responses for control nouns are also plotted for illustration. Testing for a genotype-dependent modulation of subsequent memory for P nouns relative to successful control noun encoding again reveals a significant $C$ allele-dependent enhancement of successful $\mathrm{P} v$ s. control noun encoding-related activity in the ventral striatum (Fig. 2e; Table 2). Note that the opposite genotypedependent contrast $(\mathrm{T} / \mathrm{I}>\mathrm{C} / \mathrm{I}>\mathrm{C} / \mathrm{C})$ applied to the same memory comparison, subsequent memory for $\mathrm{P}$ nouns relative to successful control noun encoding, does not reveal any significant effects at an uncorrected threshold of $P<0.001$.

We note a recent meta-analysis (Sacchet and Knutson, 2012) demonstrating that peak ventral striatal foci for studies that used smaller spatial smoothing kernels, i.e., $<6 \mathrm{~mm}$ full-width half maximum (FWHM), were more anterior than those identified for studies that used larger kernels (i.e. $>7 \mathrm{~mm}$ FWHM). Given that our original analyses employed a smoothing kernel of $8 \mathrm{~mm}$ FWHM, we repeated these
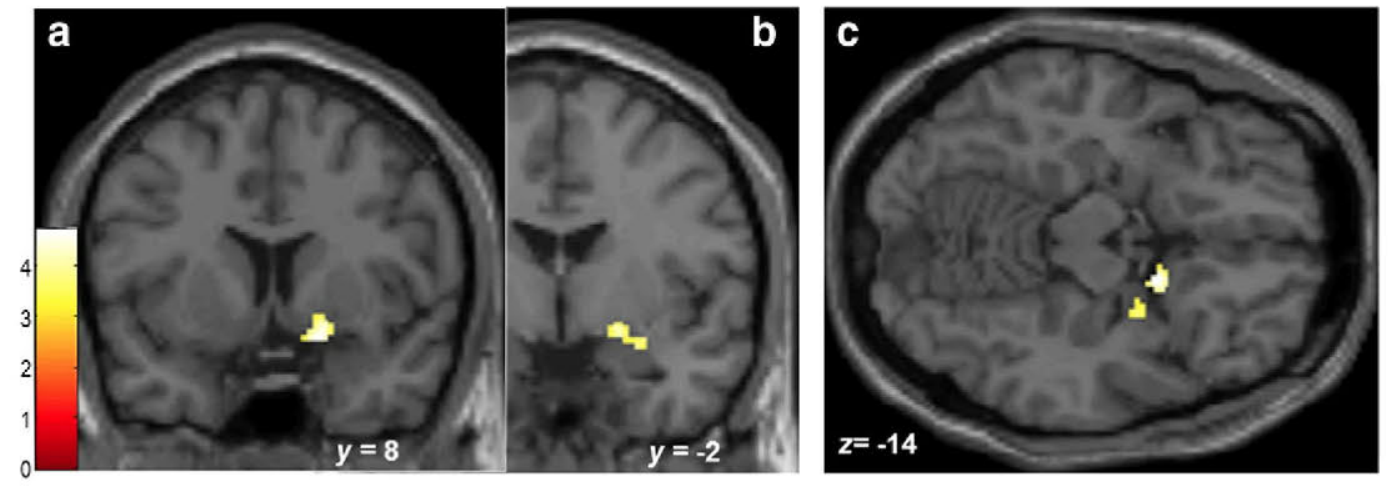

d
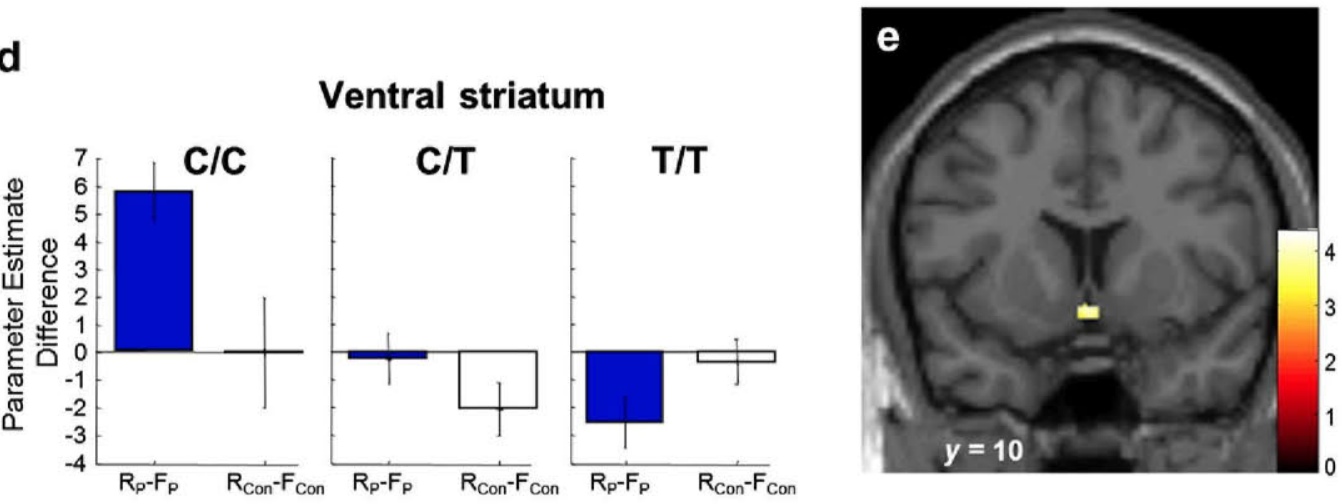

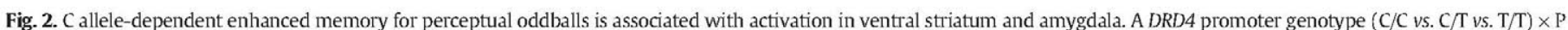

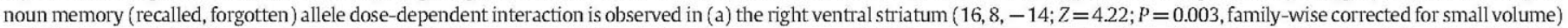

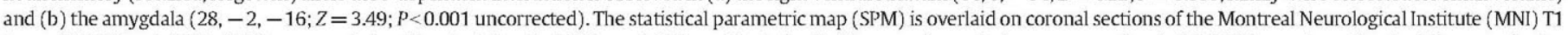

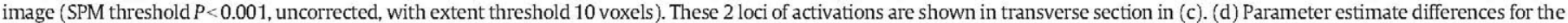

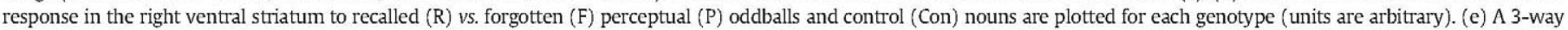

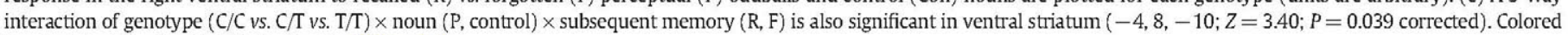
bar depicts activation T-statistic. See also Tables 1-2, 
analyses with a $6 \mathrm{~mm}$ kernel, and provide peak ventral striatal coordinates for both smoothings in Tables 1 and 2 . For the test of genotype-dependent modulation of subsequent memory for P nouns relative to successful control noun encoding, employing a $6 \mathrm{~mm}$ kernel resulted in a $2 \mathrm{~mm}$ shift anteriorly in the left ventral striatal focus (Table 2), with other foci unchanged in the antero-posterior position of their local maxima.

To ensure that the observed genotype-dependent differences in neuronal activity associated with successful $P$ noun encoding were not due to differences in recall performance, we repeated these analyses, but taking a subgroup of $\mathrm{C}$ carriers matched on performance to T/T homozygotes ( $C$ carriers were pooled for this analysis due to reduced sample size). That is, performance for each T/T subject was matched to that of the closest corresponding $\mathrm{C}$ carrier and fMRI analyses repeated, restricted to the $17 \mathrm{~T} / \mathrm{T}$ homozygotes and corresponding $17 \mathrm{C}$ carriers $(7 \mathrm{C} / \mathrm{C}$ and $10 \mathrm{C} / \mathrm{T}$ ). In this subgroup of $\mathrm{C}$ carriers, mean (s.e.m.) recall of P oddballs is $58.01 \%$ (4.72), and in the T/T subgroup 57.36\% (5.45). Recall of control nouns is $49.86 \%$ (3.44) and $50.27 \%$ (3.79) in C and T/T subgroups, respectively. Thus, enhanced P noun memory relative to control nouns is $8.14 \%$ (3.66) and 7.09\% (4.37) in C and T/T subgroups, respectively $(t(32)=$ $-0.186 P=0.853$, two-tailed). P noun subsequent memory analysis, now matched for performance, again revealed significant genotypedependent right ventral striatal and amygdala activation (Table 1). Activation in ventral striatum was again observed in the $\mathrm{P} v \mathrm{~s}$. control noun subsequent memory analysis, but at lower significance (Table 2).

Recent studies report correlations between NS scores and interindividual differences in human dopaminergic brain regions in terms of functional activation (Abler et al., 2006; Naghavi et al., 2009), dopamine ligand binding (Zald et al., 2008) and connectivity with other brain regions (Cohen et al., 2009). To ensure that the genotypedependent differences in activation we describe are independent of NS, we repeated our statistical analyses including NS as an additional covariate. Enhanced C-allele dependent responses during successful $\mathrm{P}$ noun encoding remained significant in these analyses. In view of previous reports of an association between DRD4 $-521 \mathrm{C}$ allele and NS (Munafo et al., 2008b), it is possible that the observations from neuroimaging studies on NS may be, at least in part, driven by differences in DRD4 promoter genotype.

\section{Adaptation to salience}

Animal data demonstrate that VTA responses to unexpected stimuli habituate with repeated occurrences (Ljungberg et al., 1992). fMRI studies show a similar adaptation of hippocampal responses to repeated presentations of oddball stimuli (Strange and Dolan, 2001; Yamaguchi et al., 2004). Given the critical roles of these structures in effecting memory enhancement for salient stimuli, we hypothesized that the memory enhancement for $P$ nouns would show a similar adaptation with repeated presentation of these oddballs, i.e. across successive lists. We therefore examined $P$ noun memory enhancement as a function of experimental list, limiting this analysis to the first half of the experiment (as the 17 fonts in which $P$ nouns were presented are repeated in the second half of the experiment).

Fig. 3. An adaptation of P oddball memory enhancement across successive oddball presentations is associated with adapting hippocampal and SN/VTA responses in C allele carriers, in an allele dose-dependent manner. (a) Recall performance (\%) in scanned sample for $\mathrm{P}$ oddballs, relative to control nouns, in Early (lists 1-5), Middle (6-10) and Late (11-17) lists in the first half of the experiment. The font in which $\mathrm{P}$ nouns are presented is different across the 17 lists. (b) Memory advantage for $P$ nouns show similar adaptation in the replication sample. (c-d) Activation during successful encoding of $\mathrm{P}$ oddballs, relative to contro nouns, that adapts across successive oddball presentations is observed in (c), the right hippocampus ( $38,-20,-14 ; Z=3.35 ; P=0.049$ corrected) and (d), the right SN/VTA $(4,-16,-8 ; Z=2.95 ; P=0.031$ corrected) an effect that is $C$ allele dose-dependent. Inset in (d), shows the same activation overlaid on a single-subject $\mathrm{T}^{*}$ functional image, demonstrating that the observed midbrain activation is in SN/VTA. SPM threshold for illustration $P<0.005$, uncorrected, with extent threshold of 10 voxels. Response estimates from right (e) hippocampus and (f) SN/VTA are plotted for remembered $v$ s. forgotten P oddballs relative to control nouns across Early, Middle and Late lists for each genotype. See also Table 3.
Fig. 3A demonstrates that, in the scanned sample, $\mathrm{P}$ noun memory enhancement does indeed show adaptation with repeated oddball presentation, and that this effect is expressed as a $\mathrm{C}$ allele dose-dependent effect. $\mathrm{C} / \mathrm{C}$ homozygotes show rapid adaptation of memory enhancement, C/T subjects show late adaptation, whereas P noun memory enhancement in $\mathrm{T} / \mathrm{T}$ homozygotes is constant across successive oddball presentations in the 17 different fonts (Fig. 3a). A similar pattern is observed in the replication sample (Fig. 3b). To determine the neuronal

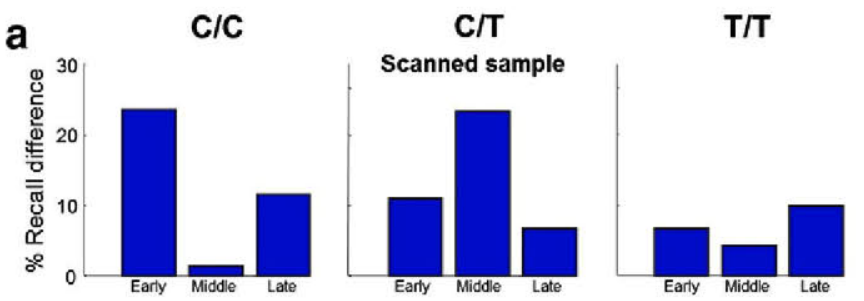

b
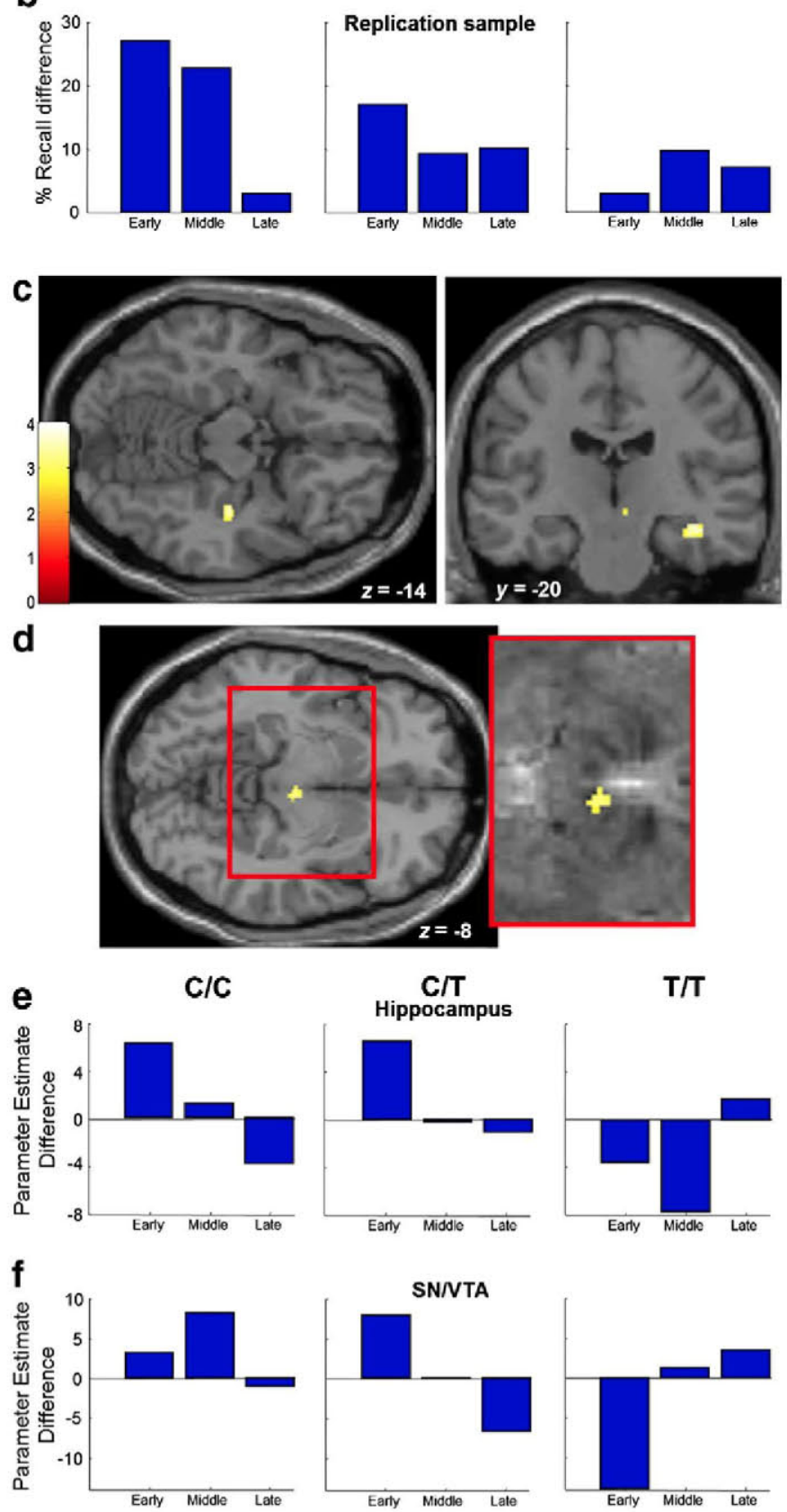
correlates of this behavioral observation, we tested for a subsequent memory effect for $\mathrm{P}$ nouns that decays across the first half of the experiment, and is expressed as a C allele dose-dependent effect. As predicted, we observe a genotype $(C / C, C / T, T / T) \times$ noun type ( $P$ noun, control) $\times$ memory $(\mathrm{R}, \mathrm{F}) \times$ repetition (linear decay) interaction in the right hippocampus (Fig. 3c) and the SN/VTA (Fig. 3d; Table 3). These effects are again observed in a repeat analysis matching $\mathrm{C}$ carriers and $\mathrm{T} / \mathrm{T}$ homozygotes on the basis of memory performance (Table 3 ). For illustration, $P$ noun recall performance (Figs. $3 a$ and $b$ ), and response estimates (Figs. $3 e$ and $\mathrm{f}$ ), in the first half of the experiment are divided into early, middle and late. Previous studies demonstrating noveltydependent activation in the dopaminergic midbrain have applied smaller smoothing kernels to their fMRI data (Bunzeck and Duzel, 2006; Guitart-Masip et al., 2010) in view of the relatively small size of these structures. We therefore repeated this analysis with data smoothed at $6 \mathrm{~mm}$ FWHM, and demonstrate the same SN/VTA activation, at slightly higher significance (Table 3).

\section{Responses in the prefrontal cortex}

The lateral prefrontal cortex (PFC) plays a critical role in novelty detection (Daffner et al., 2000; Knight, 1984) and novelty-dependent enhancements in human memory (Kishiyama et al., 2009). These findings, combined with the observations of high levels of DRD4 expression in PFC (Ariano et al., 1997; Defagot et al., 1997; Matsumoto et al., 1995) raise a possibility of $C$ allele dose-dependent PFC responses during perceptual novelty encoding. Contrary to prediction, this genotype-dependent effect was not observed, nor do we observe adaptation of prefrontal responses with repeated oddball presentation, which has been described previously (Yamaguchi et al., 2004). However, we do observe a lateral prefrontal response, in the right inferior frontal sulcus, during successful $P$ relative to control noun encoding in all genotypes (Fig. 4), suggesting a more generic novelty response in this region, independent of the $-521 C>$ T DRD4 promoter SNP.

\section{Responses to emotional oddballs}

Given that a consistent effect of DRD4 promoter polymorphism on memory enhancement was observed in both samples only for P oddballs, we focus our functional imaging analyses on this oddball type, but report here the E oddball imaging data for completeness. Preliminary analyses comparing responses to $\mathrm{E} v \mathrm{~s}$. control nouns, irrespective of whether nouns were subsequently freely recalled or forgotten, are presented in Supplementary Figs. 2 and 3. Next we performed subsequent memory analyses, identical to those for $\mathrm{P}$ nouns, except that now we tested for successful encoding-related neuronal responses to $\mathrm{E}$ oddball nouns as a function of increasing $\mathrm{C}$ allele number (with DRD4 exon III VNTR again included as a covariate of no interest). By contrast to the significant effects observed for P nouns, no brain area showed a $\mathrm{C}$ allele-dependent modulation of successful encoding-related $E$ noun responses at the threshold employed for reporting $P$ noun effects (uncorrected $P<0.001$, extent threshold 10 voxels), mirroring absent $\mathrm{E}$ noun memory effects of this genotype in the scanned sample. Testing for a genotype-dependent modulation of subsequent memory for $\mathrm{E}$ nouns relative to successful control noun encoding reveals $C$ allele-dependent enhancement of activity in several brain areas, including medial prefrontal cortex and insula (Supplementary Table 3). Employing a conjunction analysis to test for overlap in brain regions showing DRD4 promoter polymorphism-dependent modulation of successful encodingrelated activation to both perceptual and emotional oddballs, reveals effects in a widespread cortical network (Supplementary Table 4), as well as in the right amygdala.

We next examined whether neuronal responses to $\mathrm{E}$ nouns showed a similar adaptation with repeated oddball presentations, analogous to that for $P$ nouns (Figs. 3a and $b$ ). Although we did not observe a significant $(P<0.001$ uncorrected) genotype $(C / C, C / T, T / T) \times$ noun type ( $E$ noun, control $) \times$ memory $(\mathrm{R}, \mathrm{F}) \times$ repetition (linear decay) interaction in any brain area, at a liberal threshold $(P<0.05$ uncorrected) an interaction is observed in the right SN/VTA (Supplementary Table 3). The same SN/VTA region is present, corrected for small volume, testing the conjunction between the 2 oddball types for the same linear decay comparison (Supplementary Table 4).

In summary, imaging effects for DRD4 promoter polymorphismrelated modulation of $\mathrm{E}$ noun encoding were largely absent at the same statistical threshold applied to P noun analyses. This discrepancy between $E$ and $P$ noun effects might be explained by the fact that several genetic variants are known to modulate responses to emotional stimuli measured with fMRI (Hariri et al., 2002; Munafo et al., 2008a; Murphy et al., 2012; Rasch et al., 2010b; Smolka et al., 2005). Specifically, inter-individual variation in episodic memory for emotional stimuli is associated with a functionally relevant deletion variant of $A D R A 2 B$, the gene encoding the $\alpha$-2b adrenoreceptor. Carriers of this deletion variant show enhanced memory for emotional vs. neutral pictures compared with non-carriers (de Quervain et al., 2007; Rasch et al., 2009). It could therefore be the case that the variance explained by DRD4 promoter polymorphism for imaging responses to emotional memory is small compared to that of these other genotypes.

\section{Discussion}

Animal data support a model of novelty-evoked memory enhancement that is mediated by reciprocal interactions between the hippocampus and dopaminergic structures in midbrain and ventral striatum (Lisman and Grace, 2005). Our data demonstrate engagement of all these brain areas during successful encoding of contextually novel, perceptual oddballs. Critically, these effects are only observed in subjects
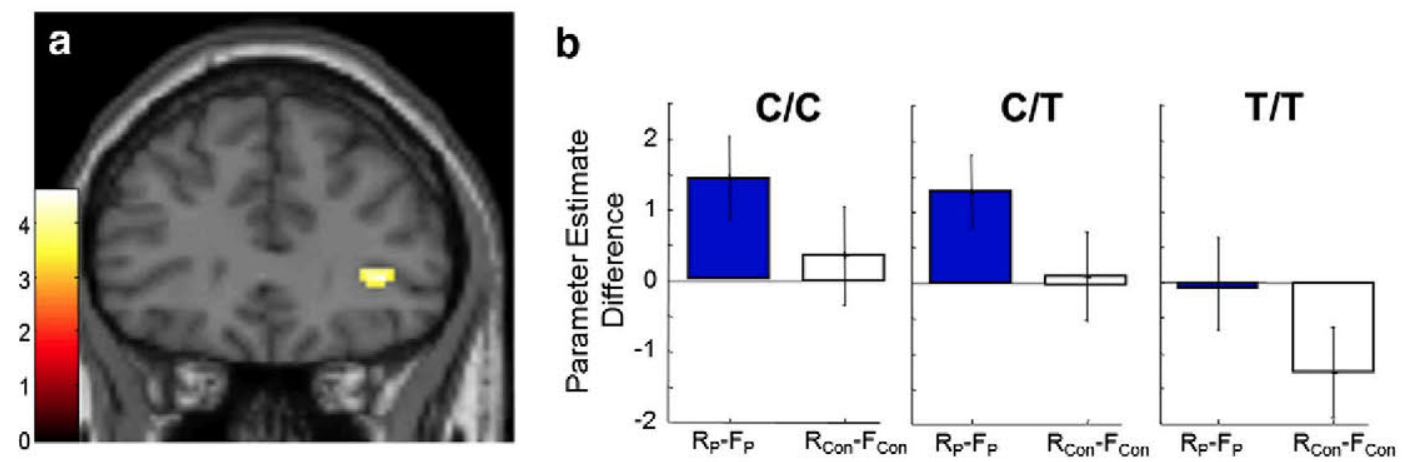

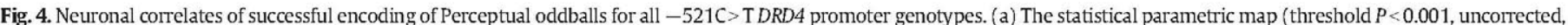

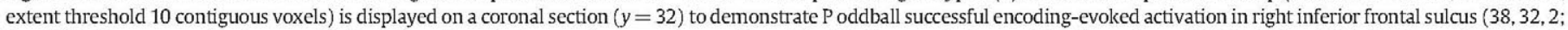

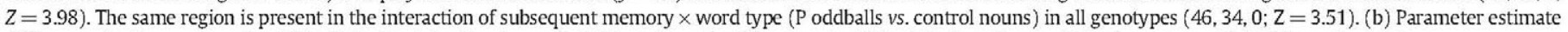
differences for the response in right inferior frontal sulcus to recalled $(R) v s$. forgotten (F) perceptual (P) oddballs and control (Con) nouns are plotted for each genotype. 
carrying the $C$ allele of the $-521 \mathrm{C}$ T DRD4 promoter polymorphism, which is mirrored by an augmented memory enhancement for salient, perceptually deviant stimuli, with both effects expressed in a $\mathrm{C}$ allele dose-dependent manner. Given statistical power considerations indicating that genotype-dependent memory effects are reliably observed at the behavioral level with a sample size greater than 100 (Rasch et al., 2010a), we complimented our memory findings in the scanned sample with a behavioral replication in a larger sample. Strikingly, the same $C$ allele dose-dependent enhancement for P noun memory is observed in both samples.

Enhanced amygdala activity is also observed in response to perceptual novelty (Figs. 2b-c), in line with previous studies, e.g. Blackford et al. (2010). This response is again dependent on $C$ allele number, with the same pattern present during $E$ noun encoding (at lower statistical significance). However, whereas patients with selective bilateral amygdala lesions performing the current task show abolished memory enhancement for emotional nouns, memory enhancement for perceptually salient nouns remains intact (Strange et al., 2003). This indicates that amygdala activity is unlikely to be an essential component of the neuronal network mediating P noun memory enhancement.

We demonstrate that $C$ allele-dependent memory enhancement for P oddballs is a dynamic process, adapting with repeated oddball presentations. This is reflected in adaptive neuronal responses in $\mathrm{C}$ carriers during successful P oddball encoding in the hippocampus and SN/VTA, in line with animal data demonstrating adaptive neuronal firing to novelty in these brain regions (Ljungberg et al., 1992; Vinogradova, 1975) and human fMRI studies showing adaptation of hippocampal responses to repeated presentations of novel oddball stimuli (Strange and Dolan, 2001; Yamaguchi et al., 2004). Adaptive SN/VTA activity was also observed during E noun encoding (at lower statistical significance), suggestive of a more generic $C$ allele-dependent modulation of responses to salience in this brain region.

Our data extend previous reports suggesting novelty-evoked activation of the dopamine system on the basis of anatomical localization of activity to dopaminergic areas (Bunzeck and Duzel, 2006; Duzel et al., 2009; Schott et al., 2004) by demonstrating that novelty-dependent activation in these regions is critically dependent on genetic variation in the DRD4 gene. Differential dopaminergic activity between genotypes in the current study is inferred from in vitro demonstration of increased DRD4 transcription levels of up to $40 \%$ in C compared with T alleles (Okuyama et al., 1999, but see Kereszturi et al., 2006; Simpson et al., 2010). With respect to a potential mechanism through which $C$ alleles confer enhanced memory for $\mathrm{P}$ nouns, it is important to note that $\mathrm{C}$ allele-dependent enhanced ventral striatal activity is observed in both performance-unmatched and matched analyses. It has been suggested (Rasch et al., 2010a) that in the case of performance-matched analyses, increased activity in the high-memory genotype may not specifically reflect the memory encoding (or retrieval) process. Following this framework (Rasch et al., 2010a), one explanation is that the effects observed at the neuronal level reflect a $\mathrm{C}$ allele-dependent enhancement of contextual novelty detection, which leads to an upregulation of memory encoding (Lisman and Grace, 2005).

An alternative approach for examining differential dopamine activity during novel stimulus encoding in the context of fMRI would be a pharmacological manipulation. However, psychopharmacological studies in healthy humans have yet to demonstrate a dopaminergic manipulation which selectively affects memory for novel stimuli. Both apomorphine, an agonist of D1 - and D2-like receptors, and haloperidol, a D2 antagonist, produce a general impairment in verbal episodic memory (Montoya et al., 2008; Rammsayer et al., 2000). The memory impairing effect of apomorphine in this previous study (Montoya et al., 2008) is, however, most likely due to apomorphine acting as a dopamine antagonist, rather than agonist, at the dose used. Although repeated administration of the dopamine precursor L-dopa has been shown to enhance learning of new words over successive days (Knecht et al., 2004), a differential benefit of L-dopa for novel vs. non-novel stimuli has yet to be shown. This is in contrast to the current data, which demonstrate selective $C$ alleledependent memory enhancement for perceptual oddballs, and no general effect of genotype on control noun memory. Selective D4 antagonists, only used experimentally in clinical studies thus far (Corrigan et al., 2004; Kramer et al., 1997), may provide further support for the critical role for D4 receptors in novelty-evoked memory enhancement.

\section{Conclusion}

Our experiment was predicated on a strong hypothesis that the dopamine system mediates enhanced memory for novel stimuli, based on animal data (Lisman and Grace, 2005) and reports of novelty-evoked activation of human dopaminergic brain areas (Bunzeck and Duzel, 2006; Duzel et al., 2009). A separate line of investigation has shown a consistent association between the $-5210 \mathrm{~T}$ DRD4 polymorphism and differential sensitivity to novelty in humans, i.e., NS personality trait (Munafo et al., 2008b). Bringing together these observations, our data demonstrate that both memory enhancement for contextually novel perceptually salient stimuli and associated activation in dopaminergic midbrain, ventral striatum and hippocampus, are critically dependent on this polymorphism.

\section{Acknowledgments}

This work was supported by the Federal Ministry of Education and Research, Deutsche Forschungsgemeinschaft (DFG KA1623/3-1 for NG, JB, RK, TRR 58 B3 for CB and Z02 to AR) and the EU (LSHM-CT-2007037286 and ERC-20IO-AdG 20100407 for CB). BS is supported by a Marie Curie Career Integration grant (FP7-PEOPLE-2011-CIG 304248) and grant SAF2011-27766 from the Spanish Ministry of Science and Innovation. We thank Nicole Döring and Frauke Fassbinder for excellent technical assistance as well as the volunteers for participating in this study.

\section{Appendix A. Supplementary data}

Supplementary data to this article can be found online at http://dx. doi.org/10.1016/j.neuroimage.2013.09.065.

\section{References}

Abler, B., Walter, H., Erk, S., Kammerer, H., Spitzer, M., 2006. Prediction error as a linear function of reward probability is coded in human nucleus accumbens. NeuroImage 31, 790-795.

Ariano, M.A., Wang, J., Noblett, K.L., Larson, E.R., Sibley, D.R., 1997. Cellular distribution of the rat D4 dopamine receptor protein in the CNS using anti-receptor antisera. Brain Res. 752, 26-34.

Benjamin, J., Li, L., Patterson, C., Greenberg, B.D., Murphy, D.L., Hamer, D.H., 1996. Population and familial association between the D4 dopamine receptor gene and measures of Novelty Seeking. Nat. Genet. 12, 81-84.

Berridge, K.C., 2007. The debate over dopamine's role in reward: the case for incentive salience. Psychopharmacology 191, 391-431.

Blackford, J.U., Buckholtz, J.W., Avery, S.N., Zald, D.H., 2010. A unique role for the human amygdala in novelty detection. Neurolmage 50,1188-1193.

Blanchard, M.M., Mendelsohn, D., Stamp, J.A., 2009. The HR/LR model: further evidence as an animal model of sensation seeking. Neurosci. Biobehav. Rev. 33, 1145-1154.

Bunzeck, N., Duzel, E., 2006. Absolute coding of stimulus novelty in the human substantia nigra/VTA. Neuron 51, 369-379.

Cloninger, R.C., 1994. The Temperament and Character Inventory (TCI): A Guide to its Development and Use. Center for Psychobiology of Personality. Washington University, St. Louis, MO.

Cloninger, C.R., Svrakic, D.M., Przybeck, T.R., 1993. A psychobiological model of temperament and character. Arch. Gen. Psychiatry 50, 975-990.

Cohen, M.X., Schoene-Bake, J.C., Elger, C.E., Weber, B., 2009. Connectivity-based segregation of the human striatum predicts personality characteristics. Nat. Neurosci. 12, 32-34.

Corrigan, M.H., Gallen, C.C., Bonura, M.L., Merchant, K.M., 2004. Effectiveness of the selective D4 antagonist sonepiprazole in schizophrenia: a placebo-controlled trial. Biol. Psychiatry $55,445-451$.

Daffner, K.R., Mesulam, M.M., Scinto, L.F., Acar, D., Calvo, V., Faust, R., Chabrerie, A., Kennedy, B., Holcomb, P., 2000. The central role of the prefrontal cortex in directing attention to novel events. Brain 123 (Pt 5), 927-939.

de Quervain, D.J., Kolassa, I.T., Ertl, V., Onyut, P.L., Neuner, F., Elbert, T., Papassotiropoulos, A., 2007. A deletion variant of the alpha2b-adrenoceptor is related to emotional memory in Europeans and Africans. Nat. Neurosci. 10, 1137-1139. 
Defagot, M.C., Malchiodi, E.L., Villar, M.J., Antonelli, M.C., 1997. Distribution of D4 dopamine receptor in rat brain with sequence-specific antibodies. Brain Res. Mol. Brain Res. $45,1-12$.

Dulawa, S.C., Grandy, D.K., Low, M.J., Paulus, M.P., Geyer, M.A., 1999. Dopamine D4 receptor-knock-out mice exhibit reduced exploration of novel stimuli. ]. Neurosci. $19,9550-9556$

Duzel, E., Bunzeck, N., Guitart-Masip, M., Wittmann, B., Schott, B.H., Tobler, P.N., 2009. Functional imaging of the human dopaminergic midbrain. Trends Neurosci. 32 321-328.

Ebstein, R.P., Novick, O., Umansky, R., Priel, B., Osher, Y., Blaine, D., Bennett, E.R., Nemanov, L Katz, M., Belmaker, R.H., 1996. Dopamine D4 receptor (D4DR) exon III polymorphism associated with the human personality trait of Novelty Seeking. Nat. Genet. 12, 78-80.

Ekelund, J., Suhonen, J., Jarvelin, M.R., Peltonen, L., Lichtermann, D., 2001. No association of the $-521 \mathrm{C} / \mathrm{T}$ polymorphism in the promoter of DRD4 with novelty seeking. Mol. Psychiatry 6, 618-619.

Franke, B., Faraone, S.V., Asherson, P., Buitelaar, J., Bau, C.H., Ramos-Quiroga, J.A., Mick, E. Grevet, E.H., Johansson, S., Haavik, J., Lesch, K.P., Cormand, B., Reif, A., 2011. The genetics of attention deficit/hyperactivity disorder in adults, a review. Mol. Psychiatry 17, 960-987.

Friston, K.J., Penny, W.D., Glaser, D.E., 2005. Conjunction revisited. Neurolmage 25 661-667.

Guitart-Masip, M., Bunzeck, N., Stephan, K.E., Dolan, R.]., Duzel, E., 2010. Contextual novelty changes reward representations in the striatum. J. Neurosci. 30, 1721-1726.

Hariri, A.R., Mattay, V.S., Tessitore, A., Kolachana, B., Fera, F., Goldman, D., Egan, M.F. Weinberger, D.R., 2002. Serotonin transporter genetic variation and the response of the human amygdala. Science $297,400-403$.

Horvitz, J.C., 2000. Mesolimbocortical and nigrostriatal dopamine responses to salien non-reward events. Neuroscience 96, 651-656.

Kabbaj, M., Devine, D.P., Savage, V.R., Akil, H., 2000. Neurobiological correlates of individual differences in novelty-seeking behavior in the rat: differential expression of stress-related molecules. J. Neurosci. 20, 6983-6988.

Kereszturi, E., Kiraly, O., Barta, C., Molnar, N., Sasvari-Szekely, M., Csapo, Z., 2006. No direc effect of the $-521 \mathrm{C} / \mathrm{T}$ polymorphism in the human dopamine D4 receptor gene promoter on transcriptional activity. BMC Mol. Biol. 7, 18.

Kishiyama, M.M., Yonelinas, A.P., Lazzara, M.M., 2004. The von Restorff effect in amnesia: the contribution of the hippocampal system to novelty-related memory enhancements. J. Cogn. Neurosci. 16, 15-23.

Kishiyama, M.M., Yonelinas, A.P., Knight, R.T., 2009. Novelty enhancements in memory are dependent on lateral prefrontal cortex. J. Neurosci. 29, 8114-8118.

Knecht, S., Breitenstein, C., Bushuven, S., Wailke, S., Kamping, S., Floel, A., Zwitserlood, P. Ringelstein, E.B., 2004. Levodopa: faster and better word learning in normal humans. Ann. Neurol. 56, 20-26.

Knight, R.T., 1984. Decreased response to novel stimuli after prefrontal lesions in man. Electroencephalogr. Clin. Neurophysiol. 59, 9-20.

Kramer, M.S., Last, B., Getson, A., Reines, S.A., 1997. The effects of a selective D4 dopamine receptor antagonist $(\mathrm{L}-745,870)$ in acutely psychotic inpatients with schizophrenia. 4 Dopamine Antagonist Group. Arch. Gen. Psychiatry 54, 567-572.

Laviolette, S.R., Lipski, W.J., Grace, A.A., 2005. A subpopulation of neurons in the medial prefrontal cortex encodes emotional learning with burst and frequency codes throug a dopamine D4 receptor-dependent basolateral amygdala input. J. Neurosci. 25, $6066-6075$.

Li, C.Y., Zhou, W.Z., Zhang, P.W., Johnson, C., Wei, L., Uhl, G.R., 2011. Meta-analysis and genome-wide interpretation of genetic susceptibility to drug addiction. BMC Genomics 12,508 .

Lisman, J.E., Grace, A.A., 2005. The hippocampal-VTA loop: controlling the entry of information into long-term memory. Neuron 46, 703-713.

Ljungberg, T., Apicella, P., Schultz, W., 1992. Responses of monkey dopamine neurons during learning of behavioral reactions. J. Neurophysiol. 67, 145-163.

Matsumoto, M., Hikosaka, O., 2009. Two types of dopamine neuron distinctly convey positive and negative motivational signals. Nature 459, 837-841.

Matsumoto, M., Hidaka, K., Tada, S., Tasaki, Y., Yamaguchi, T., 1995. Full-length cDNA cloning and distribution of human dopamine D4 receptor. Brain Res. Mol. Brain Res. 29, $157-162$.

Montoya, A., Lal, S., Menear, M., Duplessis, E., Thavundayil, J., Schmitz, N., Lepage, M 2008. Apomorphine effects on episodic memory in young healthy volunteers. Neuropsychologia 46, 292-300.

Mrzljak, L., Bergson, C., Pappy, M., Huff, R., Levenson, R., Goldman-Rakic, P.S., 1996. Localization of dopamine D4 receptors in GABAergic neurons of the primate brain. Nature $381,245-248$
Munafo, M.R., Brown, S.M., Hariri, A.R., 2008a. Serotonin transporter (5-HTTLPR) genotype and amygdala activation: a meta-analysis. Biol. Psychiatry $63,852-857$.

Munafo, M.R., Yalcin, B., Willis-Owen, S.A., Flint, J., 2008b. Association of the dopamine D4 receptor (DRD4) gene and approach-related personality traits: meta-analysis and new data. Biol. Psychiatry 63, 197-206.

Murphy, S.E., Norbury, R., Godlewska, B.R., Cowen, P.J., Mannie, Z.M., Harmer, C.J., Munafo, M.R., 2013. The effect of the serotonin transporter polymorphism (5-HTTLPR) on amygdala function: a meta-analysis. Mol. Psychiatry 18, 512-520.

Naghavi, H.R., Lind, J., Nilsson, LG., Adolfsson, R., Nyberg, L., 2009. Personality traits predict response to novel and familiar stimuli in the hippocampal region. Psychiatry Res. 173, 94-99.

Okuyama, Y., Ishiguro, H., Toru, M., Arinami, T., 1999. A genetic polymorphism in the promoter region of DRD4 associated with expression and schizophrenia. Biochem. Biophys. Res. Commun. 258, 292-295.

Powell, S.B., Paulus, M.P., Hartman, D.S., Godel, T., Geyer, M.A., 2003. RO-10-5824 is a selective dopamine D4 receptor agonist that increases novel object exploration in C57 mice. Neuropharmacology 44, 473-481.

Rammsayer, T.H., Rodewald, S., Groh, D., 2000. Dopamine-antagonistic, anticholinergic, and GABAergic effects on declarative and procedural memory functions. Brain Res. Cogn. Brain Res. 9, 61-71.

Ranganath, C., Rainer, G., 2003. Neural mechanisms for detecting and remembering novel events. Nat. Rev. Neurosci. 4, 193-202.

Rasch, B., Spalek, K., Buholzer, S., Luechinger, R., Boesiger, P., Papassotiropoulos, A., de Quervain, D.j., 2009. A genetic variation of the noradrenergic system is related to differential amygdala activation during encoding of emotional memories. Proc. Natl. Acad. Sci. U. S. A. 106, 19191-19196.

Rasch, B., Papassotiropoulos, A., de Quervain, D., 2010a. Imaging genetics of cognitive functions: focus on episodic memory. Neurolmage 53,870 .

Rasch, B., Spalek, K., Buholzer, S., Luechinger, R., Boesiger, P., de Quervain, D.J., Papassotiropoulos, A., 2010b. Aversive stimuli lead to differential amygdala activation and connectivity patterns depending on catechol-O-methyltransferase Val158Met genotype. Neurolmage 52,1712-1719.

Redgrave, P., Prescott, T.J., Gurney, K., 1999. Is the short-latency dopamine response too short to signal reward error? Trends Neurosci. 22, 146-151.

Rubinstein, M., Phillips, T.j., Bunzow, J.R., Falzone, T.L., Dziewczapolski, G., Zhang, G., Fang, Y., Larson, J.L., McDougall, JA Chester, JA Saez C, Puossley, TA Gershanik, O. Low, M.J., Grandy, D.K., 1997. Mice lacking dopamine D4 receptors are supersensitive to ethanol, cocaine, and methamphetamine. Cell 90, 991-1001.

Sacchet, M.D., Knutson, B., 2012. Spatial smoothing systematically biases the localization of reward-related brain activity. Neurolmage $66 \mathrm{C}, 270-277$

Schott, B.H., Sellner, D.B., Lauer, C.J., Habib, R., Frey, J.U., Guderian, S., Heinze, H.J., Duzel, E., 2004. Activation of midbrain structures by associative novelty and the formation of explicit memory in humans. Learn. Mem. 11, 383-387.

Simpson, J., Vetuz, G., Wilson, M., Brookes, K.J., Kent, L., 2010. The DRD4 receptor exon 3 VNTR and $5^{\prime}$ SNP variants and mRNA expression in human post-mortem brain tissue. Am. J. Med. Genet. B Neuropsychiatr. Genet. 153B, 1228-1233.

Smolka, M.N., Schumann, G., Wrase, J., Grusser, S.M., Flor, H., Mann, K., Braus, D.F., Goldman, D., Buchel, C., Heinz, A., 2005. Catechol-O-methyltransferase val158met genotype affects processing of emotional stimuli in the amygdala and prefrontal cortex. J. Neurosci. 25, 836-842.

Strange, B.A., Dolan, R.J., 2001. Adaptive anterior hippocampal responses to oddball stimuli. Hippocampus 11, 690-698

Strange, B.A., Hurlemann, R., Dolan, R.J., 2003. An emotion-induced retrograde amnesia in humans is amygdala- and beta-adrenergic-dependent. Proc. Natl. Acad. Sci. U. S. A. $100,13626-13631$

Strobel, A., Lesch, K.P., Hohenberger, K., Jatzke, S., Gutzeit, H.O., Anacker, K., Brocke, B., 2002. No association between dopamine D4 receptor gene exon III and $-521 \mathrm{C} / \mathrm{T}$ polymorphism and novelty seeking. Mol. Psychiatry 7, 537-538.

Ungless, M.A., 2004. Dopamine: the salient issue. Trends Neurosci. 27, 702-706.

Vinogradova, O.S., 1975. Functional organization of the limbic system in the process of registration of information: facts and hypotheses. In: Isaacson, R.L., Pribram, K.H. (Eds.), The Hippocampus. Plenum Press, New York, pp. 3-69.

Yamaguchi, S., Hale, L.A., D'Esposito, M., Knight, R.T., 2004. Rapid prefrontal-hippocampal habituation to novel events. J. Neurosci. 24, 5356-5363.

Zald, D.H., Cowan, R.L., Riccardi, P., Baldwin, R.M., Ansari, M.S., Li, R., Shelby, E.S., Smith, C.E., McHugo, M., Kessler, R.M., 2008. Midbrain dopamine receptor availability is inversely associated with novelty-seeking traits in humans. J. Neurosci. 28, 14372-14378.

Zink, C.F., Pagnoni, G., Martin, M.E., Dhamala, M., Berns, G.S., 2003. Human striatal response to salient nonrewarding stimuli. J. Neurosci. 23, 8092-8097. 